\title{
Dexamethasone Intravitreal Implant for Treatment of Patients With Recalcitrant Macular Edema Resulting From Irvine-Gass Syndrome
}

\author{
Marco Dutra Medeiros, ${ }^{1}$ Rafael Navarro, ${ }^{1}$ José Garcia-Arumí, ${ }^{1,2}$ Carlos Mateo, ${ }^{1}$ \\ and Borja Corcóstegui ${ }^{1}$ \\ ${ }^{1}$ Instituto de Microcirugia Ocular, Barcelona, Spain \\ ${ }^{2}$ Hospital Universitario Vall d'Hebron, Barcelona, Spain
}

Correspondence: Marco Dutra Medeiros, Instituto de Microcirurgia Ocular, Calle Josep María Lladó $\mathrm{n}^{\circ} 3$, 08035, Barcelona, Spain; marcodutramedeiros@gmail.com

Submitted: December 9, 2012 Accepted: March 30, 2013

Citation: Dutra Medeiros M, Navarro R, Garcia-Arumí J, Mateo C, Corcóstegui B. Dexamethasone intravitreal implant for treatment of patients with recalcitrant macular edema resulting from Irvine-Gass syndrome. Invest Ophthalmol Vis Sci. 2013;54:33203324. DOI:10.1167/iovs.12-11463
Purpose. To evaluate the effectiveness of a single intravitreal injection of dexamethasone implant, over 6 months in patients with recalcitrant CME due to Irvine-Gass syndrome.

Methods. Retrospective review of the medical records of nine patients with refractory macular edema (ME) due to Irvine-Gass syndrome, who underwent a single intravitreal injection of dexamethasone implant, Ozurdex, between November 2010 and January 2012, at the Instituto de Microcirurgia Ocular, Barcelona, Spain. All patients underwent a complete ophthalmic evaluation, including best-corrected visual acuity (BCVA) using standardized ETDRS charts, tonometry, fluorescein angiography, and spectral-domain optical coherence tomography with foveal thickness (FT) measurement.

REsults. The mean duration of CME before treatment with Ozurdex was 9.1 months (range, 613 months). At baseline, the mean FT was $542.22 \pm 134.78 \mu \mathrm{m}$. Mean (SD) values of FT did decrease to $350.88 \pm 98.71 \mu \mathrm{m}(P=0.001)$ at month 1 and $319.22 \pm 60.96 \mu \mathrm{m}(P=0.002)$ at month 3. Data on the 6-month follow-up showed a mild increase $398.33 \pm 127.89 \mu \mathrm{m}(P=$ 0.031). The mean (SD) change from baseline FT was $191.33 \mu \mathrm{m}$ (a decrease value of $35 \%$ ) at month 1, and $223.00 \mu \mathrm{m}$ (decrease value of $41 \%$ ) and $143.89 \mu \mathrm{m}$ (decrease value of $26 \%$ ) at month 3 and month 6 , respectively. The baseline BCVA data were $0.62 \pm 0.15$ logarithm of the minimum angle of resolution (logMAR). The mean BCVA improved to $0.47 \pm 0.21$ $\log$ MAR $(P=0.008)$ and $0.37 \pm 0.24 \operatorname{logMAR}(P=0.001)$ after month 1 and month 3 , respectively. At the last visit (6-month follow-up), the mean BCVA was $0.37 \pm 0.26$ logMAR $(P$ $=0.002)$.

Conclusions. In this study, both mean FT and mean BCVA had improved from baseline by 1 month after treatment with a dexamethasone implant, and the improvement remained statistically significant throughout the 6-month study.

Keywords: Irvine-Gass syndrome, refractory macular edema, Ozurdex
$I_{c}^{\mathrm{n}}$ n 1953, Irvine described a CME that specifically arose after cataract surgery. ${ }^{1}$ Gass and Norton subsequently studied the characteristics of the new disease entity with fluorescein angiography. ${ }^{2,3}$ Irvine-Gass syndrome is still recognized as one of the most common causes of poor visual outcome following cataract surgery. 4,5

The pathogenesis of pseudophakic CME is thought to be multifactorial. However, the major etiology appears to be inflammatory mediators that are upregulated in the aqueous and vitreous humors after surgical manipulation. Inflammation breaks down the blood-aqueous and blood-retinal-barriers, which leads to increased vascular permeability. ${ }^{6}$ Eosinophilic transudate accumulates in the outer plexiform and inner nuclear layers of the retina to create cystic spaces that coalesce to form larger pockets of fluid. ${ }^{1}$ In chronic CME, lamellar macular holes and subretinal fluid may also form.

Despite treatment options for Irvine-Gass syndrome, which include nonsteroidal anti-inflammatory drugs, corticosteroids, and acetazolamide, and new fields of clinical research including the use of antivascular endothelial growth factor agents, resistant cases of macular edema are common. ${ }^{7,8}$

Glucocorticoids such as dexamethasone exert their antiinflammatory effects by influencing multiple signal transduction pathways, including VEGF ${ }^{9-12}$ By binding to cytoplasmic glucocorticoid receptors, corticosteroids in high doses increase the activation of anti-inflammatory genes, whereas at low concentrations they have a role in the suppression of activated inflammatory genes. ${ }^{10-13}$

The dexamethasone implant (Ozurdex; Allergan, Inc., Irvine, CA) is a novel approach approved by the United States Food and Drug Administration (FDA) and by the European Union (EU) for the intravitreal treatment of macular edema (ME) after branch or central retinal vein occlusion and for the treatment of noninfectious uveitis affecting the posterior segment of the eye. ${ }^{14}$ Furthermore, clinical efficacy has been documented in other diseases, such as diabetic macular edema (DME) and persistent macular edema (ME) associated with uveitis or Irvine-Gass syndrome. ${ }^{15-20}$ Compared with published data describing other routes of administration of dexametha- 
TABLE. Baseline Demographics and Clinical Features of Study Eye

\begin{tabular}{|c|c|c|c|c|c|c|c|c|c|c|c|}
\hline $\begin{array}{c}\text { Patient } \\
\text { No. }\end{array}$ & Sex & Age, $y$ & $\begin{array}{c}\text { Duration of } \\
\text { CME Before } \\
\text { Ozurdex } \\
\text { Treatment, } \\
\text { mo }\end{array}$ & $\begin{array}{c}\text { BCVA } \\
\text { Baseline }\end{array}$ & $\begin{array}{c}\text { BCVA, } \\
\text { Month } 1\end{array}$ & $\begin{array}{c}\text { BCVA, } \\
\text { Month } 3\end{array}$ & $\begin{array}{c}\text { BCVA, } \\
\text { Month } 6\end{array}$ & $\begin{array}{c}\text { FT } \\
\text { Baseline }\end{array}$ & FT, Month 1 & FT, Month 3 & FT, Month 6 \\
\hline 1 & $\mathrm{~F}$ & 37 & 8 & 0.70 & 0.70 & 0.70 & 0.70 & 563.00 & 278.00 & 281.00 & 487.00 \\
\hline 2 & M & 83 & 12 & 0.80 & 0.50 & 0.50 & 0.50 & 662.00 & 541.00 & 371.00 & 697.00 \\
\hline 3 & F & 56 & 8 & 0.50 & 0.40 & 0.10 & 0.10 & 573.00 & 467.00 & 407.00 & 371.00 \\
\hline 4 & F & 66 & 6 & 0.80 & 0.80 & 0.70 & 0.70 & 764.00 & 363.00 & 256.00 & 310.00 \\
\hline 5 & F & 64 & 11 & 0.50 & 0.40 & 0.40 & 0.40 & 405.00 & 285.00 & 243.00 & 367.00 \\
\hline 6 & F & 65 & 7 & 0.50 & 0.10 & 0.10 & 0.00 & 601.00 & 282.00 & 273.00 & 264.00 \\
\hline 7 & M & 67 & 8 & 0.70 & 0.50 & 0.40 & 0.50 & 533.00 & 319.00 & 320.00 & 356.00 \\
\hline 8 & M & 76 & 9 & 0.40 & 0.30 & 0.10 & 0.10 & 465.00 & 381.00 & 396.00 & 401.00 \\
\hline 9 & $\mathrm{~F}$ & 92 & 13 & 0.70 & 0.50 & 0.30 & 0.30 & 314.00 & 242.00 & 326.00 & 332.00 \\
\hline
\end{tabular}

sone analogues, several results demonstrate a few advantages of this implant. ${ }^{21}$ Key features of the drug delivery system are the sustained-release formulation of the poly(lactic acid-coglycolic acid) (PLGA) matrix material, which dissolves completely in vivo, and the single-use applicator for intravitreal placement. $^{22}$

The primary purpose of this study was to evaluate the effectiveness of a single intra-vitreal injection of Ozurdex (Allergan, Inc.), over 6 months in patients with persistent CME resulting from Irvine-Gass syndrome.

\section{Subjects ANd Methods}

We retrospectively reviewed the medical records of nine patients with decreased visual acuity resulting from chronic pseudophakic CME, refractory to topical, periocular, systemic, and intravitreal treatments, who underwent a single injection of Ozurdex (Allergan, Inc.) between November 2010 and January 2012 at the Instituto de Microcirurgia Ocular, Barcelona, Spain.

Key inclusion criteria included having previously undergone a wide range of treatment options including oral carbonic anhydrase inhibitors (CAIs), topical combination therapy with nonsteroidal anti-inflammatory drugs (NSAIDs) and corticosteroids, as well as intravitreal treatment either with anti-VEGF agents or with intravitreal triamcinolone. Refractory ME was defined as persistent ME with foveal thickness (FT) of $250 \mu \mathrm{m}$ by spectral-domain optical coherence tomography (SD-OCT), lasting for at least 90 days after intravitreal anti-VEGF/steroid treatment.

Exclusion criteria included diagnosis of uncontrolled systemic disease, glaucoma, elevated IOP, and epiretinal membrane or vitreomacular traction in the study eye that could prevent improvement in visual acuity, as well as any intraocular injection, intraocular surgery, or laser treatment in the study eye within the previous 3 months. Informed consent was obtained from all patients in accordance with the Declaration of Helsinki for research involving human subjects.

At baseline, all patients underwent a complete ophthalmic evaluation, including best-corrected visual acuity (BCVA) using standardized ETDRS charts, tonometry, fluorescein angiography, and SD-OCT (Cirrus HD-OCT; Carl Zeiss Meditec, Inc., Dublin, CA) with FT measurement. Patients received a dexamethasone implant in the study eye at the baseline visit (day 1). A single-use applicator with a 22-gauge needle was used to place a dexamethasone implant in the vitreal chamber through a self-sealing scleral injection. All injections were performed in the operating room.

All patients returned to our institution at regular intervals after the surgical procedure for ocular and systemic follow-up. Patients were seen at outcome assessment study visits at day 2 and at months 1, 3, and 6. All patients were monitored for any local or systemic adverse effects during the entire study.

The primary efficacy outcome measure was the change from baseline in BVCA and in FT from baseline to month 6 . Statistical calculations were performed using the Statistical Package for Social Sciences (version 20.0; SPSS Inc., Chicago, IL). Mean changes from baseline FT and BCVA (converted to the logarithm of the minimum angle of resolution [logMAR]) were analyzed using paired $t$-tests. A 2-tailed test with an $\alpha$ level of 0.05 was used for all comparisons. We report our results herein.

\section{Results}

During the inclusion period of the study, persistent ME due to pseudophakic CME was identified in nine patients who had undergone dexamethasone implant. Baseline characteristics of patients and study eyes are listed in the Table. The patients' mean age was $67.3 \pm 15.8$ years and $66 \%$ (six of nine patients) were women.

All patients had undergone a wide range of treatments previously including oral carbonic anhydrase inhibitors (CAIs), topical combination therapy with NSAIDs and corticosteroids, as well as intravitreal treatment either with anti-VEGF agents or with intravitreal triamcinolone. The mean duration of CME before treatment with Ozurdex (Allergan, Inc.) was 9.1 months (range, 6-13 months).

At baseline, the mean FT was $542.22 \pm 134.78 \mu \mathrm{m}$. Mean (SD) values of FT did decrease to $350.88 \pm 98.71 \mu \mathrm{m}(P=$ $0.001)$ at month 1 and $319.22 \pm 60.96 \mu \mathrm{m}(P=0.002)$ at month 3. Data from the 6-month follow-up showed a mild increase $398.33 \pm 127.89 \mu \mathrm{m}(P=0.031)$. All of the FT reduction outcomes were statistically significant, with respect to baseline data $(P<0.05)$. The mean (SD) change from baseline FT was $191.33 \mu \mathrm{m}$ (a decrease value of 35\%) at month 1 , and $223.00 \mu \mathrm{m}$ (decrease value of $41 \%$ ) and $143.89 \mu \mathrm{m}$ (decrease value of 26\%) at month 3 and month 6 , respectively (Fig. 1).

Statistically significant improvement in BCVA also was seen at month 1 after treatment with a dexamethasone implant and at each subsequent follow-up visit (Fig. 2). The baseline BCVA data were $0.62 \pm 0.15 \log$ MAR. The mean BCVA improved to 


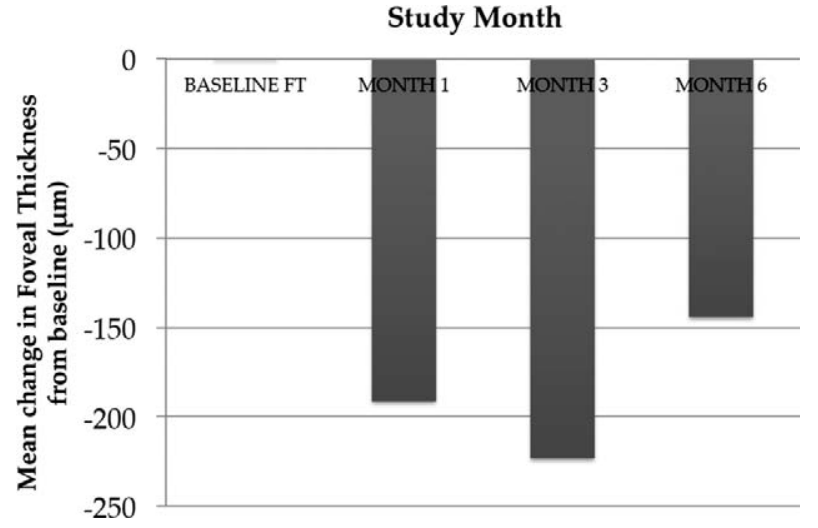

Figure 1. Mean changes from baseline foveal thickness in the study eye.

$0.47 \pm 0.21 \operatorname{logMAR}(P=0.008)$, and $0.37 \pm 0.24 \log$ MAR $(P=$ $0.001)$ after 1 and 3 months, respectively. At the last visit (6month follow-up), the mean BCVA remained the approximate value $0.37 \pm 0.26 \log$ MAR $(P=0.002)$.

No anecdotal IOP elevation was observed throughout the study. No serious ocular or systemic adverse events were observed.

\section{Discussion}

In the current study, intravitreal treatment with a dexamethasone implant safely reduced $\mathrm{ME}$ and improved visual acuity in a difficult-to-treat patient population with long-standing ME owing to Irvine-Gass syndrome.

Both mean FT and mean BCVA had improved from baseline by 1 month after treatment with a dexamethasone implant, and the improvement remained statistically significant throughout the 6-month study. The peak effectiveness of dexamethasone implants was seen at 3 months after injection, when mean FT had decreased by $41 \%$, and the mean BCVA improved to 0.37 $\pm 0.24 \log$ MAR from baseline.

So far, there are only a few controlled clinical trials that have evaluated treatments for ME associated with Irvine-Gass syndrome, including one on the use of vitrectomy for chronic aphakic CME. ${ }^{23}$ Intravitreal triamcinolone (TA) has been widely studied in DME and retinal vein occlusion, but data are lacking supporting its use in pseudophakic CME. In 2003, Benhamou et al. reported the first study using $8 \mathrm{mg}$ intravitreal triamcinolone to treat three cases of refractory chronic pseudophakic CME and showed that FT and visual acuity improved, but the effects were transient. ${ }^{24}$ Other case series also report transient benefits using $4 \mathrm{mg}$ intravitreal triamcinolone. $^{25-28}$ Clearly, one of the major limitations of intravitreal corticosteroids is the transient effect necessitating repeated injections. This problem can be overcome by the currently available sustained-release dexamethasone implant.

Most other studies have focused on the treatment of chronic pseudophakic CME that has been refractory to other treatments. In 2008, Spitzer et al. showed that $1.25 \mathrm{mg}$ intravitreal bevacizumab did not significantly improve visual outcomes in a series of 16 eyes with refractory pseudophakic CME, although there was a slight decrease in FT. ${ }^{29}$ "Triple therapy" with intravitreal triamcinolone, intravitreal bevacizumab, and topical NSAIDs has been shown to be effective as well, although the effects of the intravitreal medications were transient. ${ }^{30}$

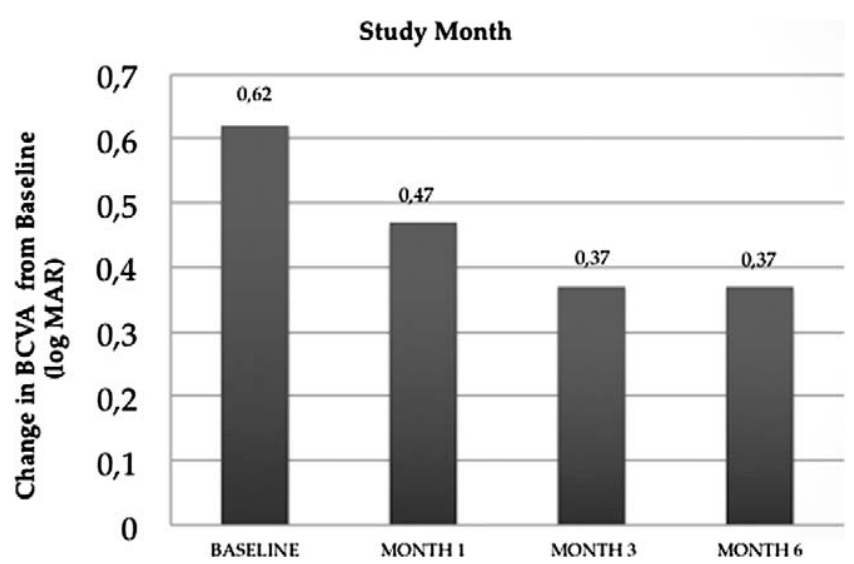

Figure 2. Mean changes from baseline BCVA in the study eye.

Sustained drug delivery systems (DDS) have been developed to address the limitation of intravitreal corticosteroid injections. Ozurdex (Allergan, Inc.) is an injectable, biodegradable intravitreal DDS that provides sustained release of preservative-free dexamethasone, a potent corticosteroid. ${ }^{31}$ In 2009, the FDA approved its use for treatment of ME secondary to retinal vein occlusions, and in 2010, it was approved for noninfectious posterior uveitis. ${ }^{14}$ A phase II study subgroup analysis investigated its efficacy in the treatment of persistent ME resulting from uveitis or pseudophakic CME. ${ }^{32}$ Twenty-seven patients with refractory pseudophakic CME were recruited and randomized to receive dexamethasone DDS $350 \mu \mathrm{g}$, dexamethasone DDS $700 \mu \mathrm{g}$, or observation. Eight patients showed at least a 10-letter improvement at day 90 and maintained the improvement at day 180. Significance testing was performed with combined data from pseudophakic CME and uveitis patients: an improvement in BCVA of at least 10 letters at day 90 was seen in $42 \%$ in the $350 \mu \mathrm{m}$ group $(P=$ $0.117)$, and $54 \%$ in the $700 \mu \mathrm{m}$ group $(P=0.029)$. Another study is currently enrolling patients for a phase II study to examine the dexamethasone DDS to treat CME and DME after cataract surgery. ${ }^{33}$

Similarly, our data are consistent with those results named above. Indeed, the dexamethasone implant resulted in sustained levels of dexamethasone and biological activity for 6 months, with peak levels of drug over the first 3 months. ${ }^{17-19}$

The target population in the current study was difficult to treat because it included severe cases of long-standing ME after cataract surgery that had failed to respond to previous wide range of treatment options including oral CAIs, topical combination therapy with NSAIDs and corticosteroids, as well as intravitreal treatment, most commonly intravitreal injection of the corticosteroid TA or the anti-VEGF therapy. The mean duration of CME before treatment with Ozurdex (Allergan, Inc.) was 9.1 months (range, 6-13 months).

The treatment approach for ME has been dynamic. Over the past decades, corticosteroids have raised interest in the treatment of ME because of their anti-inflammatory effects and because they inhibit VEGF synthesis and reduce vascular permeability. Nonetheless, due safety concerns ${ }^{34}$ (i.e., the risk of IOP elevation and cataract progression) in recent years, the use of corticosteroids has been drastically reduced in most developed countries. Recently, the safety profile of Ozurdex (Allergan, Inc.), which is currently approved for the treatment of retinal vein occlusion, was addressed in GENEVA study. ${ }^{14}$ In the present series, no major side effects were recorded. 
Dexamethasone intravitreal implants were well tolerated and no anecdotal IOP elevation was observed throughout the study.

Four patients had undergone a pars plana vitrectomy before entering the current study. The improvement in FT and BCVA seen in this sample was similar to the improvement seen in the remaining nonvitrectomized patients. The results of the present study demonstrate that a dexamethasone implant may be an effective treatment for pseudophakic CME in vitrectomized patients, even in severe cases that have failed to respond to previous therapies. Our data are consistent with those from a recent analysis in an earlier publication addressing this issue. ${ }^{19}$ Advantages of dexamethasone implants over intravitreal TA in vitrectomized eyes include a longer duration of action and absence of postinjection "visual clouding" and floater-like symptoms.

So far, a literature review indicates that the single-injection of the implant is well tolerated and produces meaningful improvements in ME and visual acuity that persist through 6 months. However, future longer-term trials are needed to evaluate the efficacy and safety data in patients who receive multiple injections.

Our study has several limitations-being a short-term, openlabel, uncontrolled, and retrospective-that preclude any estimation of the long-term efficacy or safety of intravitreal Ozurdex (Allergan, Inc.). A significant limitation of this study is that it included only a relatively small number of patients (nine eyes) and was not powered to show statistically significant between-group differences.

Nevertheless, the findings of the present study suggest that the dexamethasone implant Ozurdex (Allergan, Inc.) may be a promising new treatment option for patients with recalcitrant macular edema resulting from Irvine-Gass syndrome and support further investigation of its clinical value in this patient population.

\section{Acknowledgments}

Disclosure: M. Dutra Medeiros, None; R. Navarro, None; J. Garcia-Arumí, None; C. Mateo, None; B. Corcóstegui, None

\section{References}

1. Irvine SR. A newly defined vitreous syndrome following cataract surgery. Am J Ophthalmol. 1953;36:599-619.

2. Gass JDM, Norton EWD. Fluorescein studies of patients with macular edema and papilledema following cataract extraction. Trans Am Ophthalmol Soc. 1966;64:232-249.

3. Gass JDM, Norton EWD. Cystoid macular edema and papilledema following cataract extraction. Arch Ophthalmol. 1966;76:646-661.

4. Flach AJ. The incidence, pathogenesis and treatment of cystoid macular edema following cataract surgery. Trans Am Ophthalmol Soc. 1998;96:557-634.

5. Yonekawa Y, Kim IK. Pseudophakic cystoid macular edema. Curr Opin Ophthalmol. 2012;23:26-32.

6. Benitah NR, Arroyo JG. Pseudophakic cystoid macular edema. Int Opbthalmol Clin. 2010;50:139-153.

7. Shelsta HN, Jampol LM. Pharmacologic therapy of pseudophakic cystoid macular edema: 2010 update. Retina. 2011;31: 4-12.

8. Sivaprasad S, Bunce C, Wormland R. Non-steroidal antiinflammatory agents for cystoid macular edema following cataract surgery: a systematic review. Br J Ophthalmol. 2005; 89:1420-1422.

9. Abraham SM, Lawrence T, Kleiman A, et al. Antiinflammatory effects of dexamethasone are partly dependent on induction of dual specificity phosphatase 1. J Exp Med. 2006;203:18831889.

10. Barnes PJ. Corticosteroid effects on cell signalling. Eur Respir J. 2006;27:413-426.

11. Saklatvala J. Glucocorticoids: do we know how they work? Arthritis Res. 2002;4:146-150.

12. Walker BR. Glucocorticoids and cardiovascular disease. Eur J Endocrinol. 2007;157:545-559.

13. Nauck M, Karakiulakis G, Perruchoud AP, et al. Corticosteroids inhibit the expression of the vascular endothelial growth factor gene in human vascular smooth muscle cells. Eur $J$ Pharmacol. 1998;341:309-315.

14. Haller JA, Bandello F, Belfort R Jr, et al.; Ozurdex GENEVA Study Group. Randomized, sham-controlled trial of dexamethasone intravitreal implant in patients with macular edema due to retinal vein occlusion. Ophthalmology. 2010;117:1134-1146.

15. Haller JA, Kuppermann BD, Blumenkranz MS, et al.; Dexamethasone DDS Phase II Study Group. Randomized controlled trial of an intravitreous dexamethasone drug delivery system in patients with diabetic macular edema. Arch Ophthalmol. 2010;128:289-296.

16. Boyer DS, Faber D, Gupta S, et al.; Ozurdex Champlain Study Group. Dexamethasone intravitreal implant for treatment of diabetic macular edema in vitrectomized patients. Retina. 2011;31:915-923.

17. Chang-Lin JE, Attar M, Acheampong AA, et al. Pharmacokinetics and pharmacodynamics of a sustained-release dexamethasone intravitreal implant. Invest Ophthalmol Vis Sci. 2011;52: 80-86.

18. Saraiya NV, Goldstein DA. Dexamethasone for ocular inflammation. Expert Opin Pharmacother. 2011;12:1127-1131.

19. Chang-Lin JE, Burke JA, Peng Q, et al. Pharmacokinetics of a sustained-release dexamethasone intravitreal implant in vitrectomized and nonvitrectomized eyes. Invest Ophthalmol Vis Sci. 2011;52:4605-4609.

20. Lowder C, Belfort R Jr, Lightman S, et al.; Ozurdex HURON Study Group. Dexamethasone intravitreal implant for noninfectious intermediate or posterior uveitis. Arch Ophthalmol. 2011;129:545-553.

21. Kodama M, Numaga J, Yoshida A. Effects of a new dexamethasone-delivery system (Surodex) on experimental intraocular inflammation models. Graefes Arch Clin Exp Ophthalmol. 2003;241:927-933.

22. Sivaprasad S, McCluskey P, Lightman S. Intravitreal steroids in the management of macular oedema. Acta Ophthalmol Scand. 2006;84:722-733.

23. Fung WE. Vitrectomy for chronic aphakic cystoid macular edema: results of a national, collaborative, prospective, randomized investigation. Ophthalmology. 1985;92:11021111.

24. Benhamou N, Massin P, Haouchine B. Intravitreal triamcinolone for refractory pseudophakic macular edema. Am J Ophthalmol. 2003;135:246-249.

25. Conway MD, Canakis C, Livir-Rallatos C. Intravitreal triamcinolone acetonide for refractory chronic pseudophakic cystoid macular edema. J Cataract Refract Surg. 2003;29:27-33.

26. Deuter CM, Gelisken F, Stubiger N. Successful treatment of chronic pseudophakic macular edema (Irvine-Gass syndrome) with interferon alpha: a report of three cases. Ocul Immunol Inflamm. 2011;19:216-218.

27. Wu L, Arevalo JF, Hernandez-Bogantes E. Intravitreal infliximab for refractory pseudophakic cystoid macular edema: results of the Pan-American Collaborative Retina Study Group. Int Ophthalmol. 2012;32:235-243.

28. Steinert RF, Wasson PJ. Neodymium: YAG laser anterior vitreolysis for Irvine-Gass cystoid macular edema. J Cataract Refract Surg. 1989;15:304-307. 
29. Spitzer MS, Ziemssen F, Yoeruek E. Efficacy of intravitreal bevacizumab in treating postoperative pseudophakic cystoid macular edema. J Cataract Refract Surg. 2008;34:70-75.

30. Warren KA, Bahrani H, Fox JE. NSAIDs in combination therapy for the treatment of chronic pseudophakic cystoid macular edema. Retina. 2010;30:260-266.

31. London NJ, Chiang A, Haller JA. The dexamethasone drug delivery system: indications and evidence. Adv Ther. 2011;28: 351-366.

32. Williams GA, Haller JA, Kuppermann BD. Dexamethasone posterior-segment drug delivery system in the treatment of macular edema resulting from uveitis or Irvine-Gass syndrome. Am J Ophthalmol. 2009;147:1048-1054.

33. Khurana R. Ozurdex for combined pseudophakic cystoid macular edema and diabetic macular edema after cataract surgery. US NIH. 2012. Available at: http://clinicaltrials.gov/ ct2/show/NCT01284478. Accessed November 21, 2012.

34. Diabetic Retinopathy Clinical Research Network (DRCR.net), Beck RW, Edwards AR, et al. Three-year follow-up of a randomized trial comparing focal/grid photocoagulation and intravitreal triamcinolone for diabetic macular edema. Arch Ophthalmol. 2009;127:245-251. 